\title{
Experimental study on noise reduction and performance enhancement for internal combustion engines
}

\author{
Ioan Radu Şugar ${ }^{1}$ and Mihai Banica ${ }^{1 *}$ \\ ${ }^{1}$ Technical University of Cluj-Napoca, Faculty of Engineering, Victor Babes 62A, 430083, Baia Mare, \\ Romania
}

\begin{abstract}
As the number of cars increases and large cities become more and more crowded, noise reduction becomes more and more important. The decrease of the fuel consumption and the increase of power to the same cylindrical capacity are always current topics. This paper's aim is to bring a contribution to solving these problems. The proposed solution consists in the use of ceramic materials in the design of the combustion chamber.
\end{abstract}

\section{Introduction}

Although internal combustion engines were invented in the middle of the 19th century, they went on for more than half a century before imposing themselves in front of electric motors to equip onto cars. This is largely due to Henry Ford's innovative ideas put into practice in $1913[1,3,5]$.

With global warming and the depletion of fossil fuel reserves after almost a hundred years, the debate between electrical and internal combustion cars has been resumed. On the other hand, on a larger scale, biofuel is required in fuelling internal combustion engines. The cars equipped with electric motors seem ideal at first sight as they are more friendly to the environment. According to some researchers in the field, manufacturing of electric batteries represents approx. $80 \%$ of that made by an Otto engine car. Also, recycling the batteries is still an unsolved problem due to their lack of standardization. Internal combustion engines will have a relatively long-life equipping hybrid car $[2,7,8]$.

Internal combustion engines will still represent the solution for the propulsion of trucks, military equipment, ships etc [4, 5]. Improving the performance indices of internal combustion engines remains an ongoing research topic to increase the performance of internal combustion engines, the use of new materials in general and the use of ceramic materials may be a solution [3, 9].

"Advanced ceramics" represents a group of structural and functional materials, with superior characteristics to the classical variants. They are designed for special fields of use, where they offer a higher performance compared to the metallic or polymeric materials. Advanced ceramics intervene in applications where the latter classes of products reach their limits $[6,9]$.

\footnotetext{
* Corresponding author: Mihai.Banica@cunbm.utcluj.ro
} 
The new class of "advanced ceramic" materials, which entered the market in the 20th century includes much more pure material systems, with specially processed compounds. They were developed mainly for structural and electronic applications. Advanced ceramics are distinguished by their high chemical purity and high values of use characteristics $[6,9]$.

In the last 25 years, new structural materials, such as ceramics, polymers and composites, have caused revolutionary changes in the field of materials engineering. With advanced ceramics and composites, the concepts of materials and structures taken together have led to a new concept of integrated design. Each projected material consolidates the discrete and functional parts into one, multifunctional structure, which leads to the highest efficiency of material use and the lowest costs $[6,9]$.

The innovative technological ceramics extend their use in the engineering of applications that exploit their mechanical properties. All applications for cutting-edge technologies require components that have high mechanical and wear resistance, along with high toughness, high breaking resistance through ballistic impact. They add tenacity, chemical inertia and ability to work at high temperatures. In some cases, they may have special functions: electrical, magnetic, optical or chemical-biological $[6,9]$.

\section{Equipment and methods}

In the early 1980s, the Japanese researchers from TOYOTA MOTORS made a 100\% ceramic engine. The idea was abandoned due to its modest reliability of only 250 hours of operation.

Currently, composite materials are used for building engines: they contain a metal part and a ceramic part. The metal part provides mechanical strength, and the ceramic one achieves the thermal barrier in the most thermally demanded areas. The ceramic part was made by sintering.

Ceramic coatings have been known to implement these directions: isolation chamber, isolating the exhaust path, isolating hot components. Our research focused on isolating the piston head with $\mathrm{Al}_{2} \mathrm{O}_{3}$. Since the main mechanical stress is given by the gas pressure, the technical solution chosen was adhesive bonding of the ceramic crown on the piston surface.

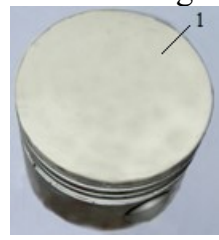

Fig. 1. Insulated piston with ceramic crown: 1-ceramic crown

The model of the experimental stand is presented in the following figure:

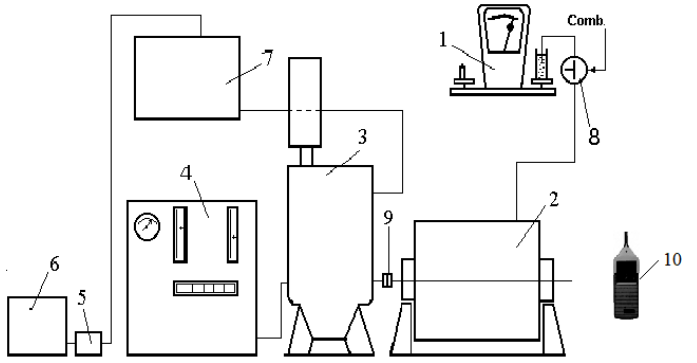

1-mass measurement of fuel consumption; 2-engine 810-99; 3-hydraulic brake with electromotor and analogue apparatus for determining the motor moment; 4-control block with digital display of coolant temperature, oil temperature and pressure and crankshaft speed; 5-electric pump; 6 and 7-coolant basin; 8-way three-way valve; 9-cardan coupling; 10-Digital Sound Level Meter

Fig. 2. Model of the experimental stand. 
For the experimental part, an 810-99 engine was used with the following characteristics: SIE motor type; 8.5:1 compression ratio; Cylinder diameter $73 \mathrm{~mm}$; Piston stroke $77 \mathrm{~mm}$; Total cylinder $1289 \mathrm{~cm}^{3}$; Maximum power $39.72 \mathrm{~kW}$ at $5250 \mathrm{rpm}$; Maximum torque $95 \mathrm{Nm}$ at 3.000rpm; Number of times 4; Positioning of cylinders: Line.

The characteristic parameters and admissible measurement errors are presented in Table 1 and general conditions for carrying out the experiment are summarized in Table 2.

Table 1. Characteristic parameters and admissible measurement errors.

\begin{tabular}{|l|c|c|c|}
\hline \multicolumn{1}{|c|}{ Parameter } & Symbol & $\begin{array}{c}\text { Unit of } \\
\text { measurement }\end{array}$ & $\begin{array}{c}\text { Admissible } \\
\text { measurement errors }\end{array}$ \\
\hline Actual motor momentum & $\mathrm{M}_{\mathrm{e}}$ & $\mathrm{N} \cdot \mathrm{m}$ & $\pm 1 \%$ \\
\hline Brake indication & $\mathrm{F}$ & $\mathrm{N}$ & $\pm 1 \%$ \\
\hline Arm length from the weighing device & $\mathrm{l}$ & $\mathrm{m}$ & - \\
\hline Drive shaft speed & $\mathrm{n}$ & $\mathrm{rpm}$ & $\pm 0,5 \%$ \\
\hline Hourly fuel consumption & $\mathrm{C}, \mathrm{C}_{\mathrm{h}}$ & $1 / \mathrm{h}, \mathrm{kg} / \mathrm{h}$ & $\pm 1 \%$ \\
\hline Hourly air consumption & $\mathrm{C}_{\mathrm{a}}$ & $\mathrm{m}^{3} / \mathrm{h}, \mathrm{kg} / \mathrm{h}$ & $\pm 2 \%$ \\
\hline Air temperature allowed & $\mathrm{T}_{\text {aer, }}, \mathrm{T}_{\mathrm{o}}$ & $\mathrm{K}$ & $\pm 2 \mathrm{~K}$ \\
\hline Coolant temperature & $\mathrm{T}_{\mathrm{apa}}, \mathrm{T}_{\mathrm{r}}$ & $\mathrm{K}$ & $\pm 2 \mathrm{~K}$ \\
\hline Oil temperature at the measuring site & $\mathrm{T}_{\mathrm{u}}$ & $\mathrm{K}$ & $\pm 2 \mathrm{~K}$ \\
\hline Temperature of exhaust gases & $\mathrm{T}_{\mathrm{ge}}$ & $\mathrm{K}$ & $\pm 5 \mathrm{~K}$ \\
\hline Fuel temperature & $\mathrm{T}_{\text {comb }}$ & $\mathrm{K}$ & $\pm 2 \mathrm{~K}$ \\
\hline Atmospheric pressure (barometric) & $\mathrm{p}_{\text {atm }},\left(\mathrm{p}_{\mathrm{b}}\right)$ & $\mathrm{bar},(\mathrm{KPa})$ & $\pm 0,1 \mathrm{KPa}$ \\
\hline Oil pressure at the indicated location & $\mathrm{p}_{\mathrm{u}}$ & $\mathrm{KPa}$ & $\pm 10 \mathrm{KPa}$ \\
\hline Pressure or depression in the exhaust system & $\mathrm{p}_{\text {sist }}$ ev. & $\mathrm{bar}, \mathrm{KPa}$ & $\pm 0,1 \mathrm{KPa}$ \\
\hline Pressure drop on the air filter & $\mathrm{p}_{\text {filtru }}$ & $\mathrm{KPa}$ & $\pm 0,05 \mathrm{KPa}$ \\
\hline Depression at the admission gallery & $\mathrm{p}_{\mathrm{adm}}$ & $\mathrm{bar}, \mathrm{KPa}$ & $\pm 0,05 \mathrm{KPa}$ \\
\hline Depression of control of the advance & $\mathrm{p}_{\mathrm{av}}$ & $\mathrm{KPa}$ & $\pm 1 \%$ \\
\hline Ignition advance angle & $\beta_{\mathrm{s}}$ & $\mathrm{RAC}$ & $\pm 1 \%$ \\
\hline Time & $\mathrm{t}$ & $\mathrm{s}$ & $\pm 0,1 \mathrm{~s}$ \\
\hline
\end{tabular}

Table 2. General conditions.

\begin{tabular}{|l|l|l|}
\hline \multirow{2}{*}{ Engine type } & \multicolumn{2}{|c|}{ Standard / Modified } \\
\cline { 2 - 3 } & \multicolumn{2}{|c|}{ Engine: $810-99+$ Carburettor: $32 I R M-A$} \\
\hline Purpose of the test & Determining the effective power \\
\hline \multirow{2}{*}{ Test conditions } & Air temperature: $28^{\circ} \mathrm{C}$ & Atmospheric pressure: $740 \mathrm{mmHg}$ \\
\cline { 2 - 3 } & Coolant temperature: $83 \div 92^{\circ} \mathrm{C}$ & Oil temperature: $93 \div 117^{\circ} \mathrm{C}$ \\
\hline Fuel and lubricant & Petrol: PREMIUM II (STAS 176-80) & Oil: M20W40 (STAS 9171-80) \\
\hline
\end{tabular}

\section{Results and discussions}

The values of measured parameters (Braking force $(F)$, Fuel mass consumed $(m)$ and Sound pressure level $(S P L))$ are presented in the Table 3.

\subsection{Calculated parameters}

Effective power $\left(P_{e}\right)$ :

$$
P_{e}=\frac{F \cdot n}{13600} \quad[k W]
$$


Actual specific consumption $\left(C_{e}\right)$ :

$$
C_{e}=3,6 \frac{\mathrm{m}}{\tau \cdot P_{e}}\left[\frac{\mathrm{kg}}{\mathrm{h}}\right]
$$

Actual efficiency $\left(\eta_{e}\right)$ :

$$
\eta_{e}=\frac{3600}{C_{e} \cdot Q_{i}}=\frac{3600}{C_{e} \cdot 43890}
$$

where: $Q_{i}$ is the calorific value of the fuel used.

The values of calculated parameters (Effective power $\left(P_{e}\right)$, Actual specific consumption $\left(C_{e}\right)$ and Actual efficiency $\left.\left(\eta_{e}\right)\right)$ are presented in the Table 4.

\begin{tabular}{|c|c|c|c|c|c|c|c|}
\hline \multirow[t]{3}{*}{ rpm } & \multirow{2}{*}{$\begin{array}{l}\text { Time } \\
\text { of } \\
\text { trial } \\
(\tau)\end{array}$} & \multicolumn{3}{|c|}{ Standard engine } & \multicolumn{3}{|c|}{ Modified engine } \\
\hline & & $\begin{array}{l}\text { Braking } \\
\text { force } \\
(F)\end{array}$ & $\begin{array}{l}\text { Fuel mass } \\
\text { consumed } \\
(\mathrm{m})\end{array}$ & $\begin{array}{c}\text { Sound } \\
\text { pressure level } \\
(S P L)\end{array}$ & $\begin{array}{l}\text { Braking } \\
\text { force } \\
(F)\end{array}$ & $\begin{array}{l}\text { Fuel mass } \\
\text { consumed } \\
(\mathrm{m})\end{array}$ & $\begin{array}{c}\text { Sound } \\
\text { pressure level } \\
(S P L)\end{array}$ \\
\hline & {$[s]$} & {$[N]$} & [g] & {$[d B]$} & {$[N]$} & [g] & {$[d B]$} \\
\hline 1500 & 32 & 115,6 & 40,35 & 61,0 & 118,2 & 39,28 & 58,0 \\
\hline 2000 & 30 & 121,0 & 45,38 & 62,0 & 125,3 & 44,53 & 60,0 \\
\hline 2250 & 24 & 125,5 & 41,25 & 63,0 & 128,3 & 39,62 & 61,0 \\
\hline 2625 & 25 & 130,3 & 52,22 & 64,5 & 133,8 & 50,75 & 62,5 \\
\hline 3000 & 22 & 132,4 & 54,26 & 67,0 & 135,2 & 52,67 & 64,0 \\
\hline 3375 & 19 & 128,1 & 50,33 & 67,5 & 132,0 & 48,75 & 65,5 \\
\hline 3750 & 18 & 125,3 & 50,79 & 68,0 & 128,3 & 48,82 & 66,5 \\
\hline 4125 & 16 & 121,0 & 47,63 & 69,0 & 124,3 & 45,74 & 67,0 \\
\hline 4500 & 15 & 115,3 & 47,37 & 71,0 & 117,7 & 45,11 & 68,0 \\
\hline 4875 & 15 & 109,0 & 49,82 & 72,5 & 110,0 & 47,64 & 68,5 \\
\hline 5250 & 14 & 101,3 & 48,21 & 74,0 & 102,9 & 46,34 & 71,5 \\
\hline 5500 & 13 & 91,2 & 44,22 & 75,0 & 94,9 & 43,66 & 73,0 \\
\hline
\end{tabular}

Table 3. The values of the measured parameters.

Table 4. The values of the calculated parameters.

\begin{tabular}{|c|c|c|c|c|c|c|}
\hline \multirow{2}{*}{ rpm } & \multicolumn{3}{|c|}{ Standard engine } & \multicolumn{3}{c|}{ Modified engine } \\
\cline { 2 - 7 } & $\begin{array}{c}\text { Effective } \\
\text { power } \\
\left(P_{e}\right)\end{array}$ & $\begin{array}{c}\text { Actual specific } \\
\text { consumption } \\
\left(C_{e}\right)\end{array}$ & $\begin{array}{c}\text { Actual } \\
\text { efficiency } \\
\left(\eta_{e}\right)\end{array}$ & $\begin{array}{c}\text { Effective } \\
\text { power } \\
\left(P_{e}\right)\end{array}$ & $\begin{array}{c}\text { Actual specific } \\
\text { consumption } \\
\left(C_{e}\right)\end{array}$ & $\begin{array}{c}\text { Actual } \\
\text { efficiency } \\
\left(\eta_{e}\right)\end{array}$ \\
\cline { 2 - 7 } & {$[\mathrm{kW}]$} & {$[\mathrm{kg} / \mathrm{kWh}]$} & {$[\%]$} & {$[\mathrm{kW}]$} & {$[\mathrm{kg} / \mathrm{kWh}]$} & {$[\%]$} \\
\hline 1500 & 12,75 & 0,356 & 23,04 & 13,04 & 0,339 & 24,20 \\
\hline 2000 & 17,79 & 0,306 & 26,80 & 18,43 & 0,290 & 28,28 \\
\hline 2250 & 20,76 & 0,298 & 27,52 & 21,23 & 0,280 & 29,29 \\
\hline 2625 & 25,15 & 0,299 & 27,43 & 25,83 & 0,283 & 28,98 \\
\hline 3000 & 29,21 & 0,304 & 26,98 & 29,82 & 0,289 & 28,38 \\
\hline 3375 & 31,79 & 0,300 & 27,34 & 32,76 & 0,282 & 29,09 \\
\hline 3750 & 34,55 & 0,294 & 27,90 & 35,38 & 0,276 & 29,72 \\
\hline 4125 & 36,70 & 0,292 & 28,09 & 37,70 & 0,273 & 30,05 \\
\hline 4500 & 38,15 & 0,298 & 27,52 & 38,94 & 0,278 & 29,50 \\
\hline 4875 & 39,07 & 0,306 & 26,80 & 39,43 & 0,290 & 28,28 \\
\hline 5250 & 39,10 & 0,317 & 25,87 & 39,72 & 0,300 & 27,34 \\
\hline 5500 & 36,88 & 0,332 & 24,71 & 38,38 & 0,315 & 26,04 \\
\hline
\end{tabular}




\subsection{Statistical analysis}

Comparative analysis of functional parameter values of the two engines obtained in the experiment is presented below:

Table 5. Analysis of functional parameter values.

\begin{tabular}{|c|c|c|c|c|c|c|c|c|c|c|c|c|}
\hline \multirow[t]{2}{*}{ rpm } & $\begin{array}{c}\text { Standard } \\
\text { engine }\end{array}$ & $\begin{array}{c}\text { Modified } \\
\text { engine }\end{array}$ & \multirow{2}{*}{$\begin{array}{c}\text { Relative } \\
\text { increase } \\
\left(\boldsymbol{P}_{e}\right) \\
{[\%]}\end{array}$} & $\begin{array}{c}\text { Standard } \\
\text { engine }\end{array}$ & $\begin{array}{c}\text { Modified } \\
\text { engine }\end{array}$ & \multirow{2}{*}{$\begin{array}{c}\text { Relative } \\
\text { decrease } \\
\left(C_{e}\right) \\
{[\%]}\end{array}$} & \multirow{2}{*}{\begin{tabular}{|c|}
$\begin{array}{c}\text { Standard } \\
\text { engine }\end{array}$ \\
$\eta_{\mathrm{e}}^{*}$ \\
{$[\%]$}
\end{tabular}} & \multirow{2}{*}{\begin{tabular}{|c|}
$\begin{array}{c}\text { Modified } \\
\text { engine }\end{array}$ \\
$\eta_{\mathrm{e}}{ }^{*}$ \\
{$[\%]$}
\end{tabular}} & \multirow{2}{*}{$\begin{array}{c}\text { Relative } \\
\text { increase } \\
\left(\eta_{e}\right)^{*} \\
{[\%]}\end{array}$} & \multirow{2}{*}{\begin{tabular}{|c|}
$\begin{array}{c}\text { Standard } \\
\text { engine }\end{array}$ \\
SPL \\
{$[\mathrm{dB}]$}
\end{tabular}} & \multirow{2}{*}{ 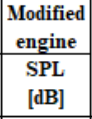 } & \multirow{2}{*}{$\begin{array}{c}\text { Relative } \\
\text { decrease } \\
\text { (SPL) } \\
{[\%]}\end{array}$} \\
\hline & $\begin{array}{c}P_{e} \\
{[k W]}\end{array}$ & $\begin{array}{c}P_{e} \\
{[k W]}\end{array}$ & & $\begin{array}{c}\mathrm{Ce} \\
{[\mathrm{kg} / \mathrm{kWh}]}\end{array}$ & $\begin{array}{c}\mathrm{Ce} \\
{[\mathrm{kg} / \mathrm{kWh}]}\end{array}$ & & & & & & & \\
\hline 1500 & 12,75 & 13,04 & 2,25 & 0,356 & 0,339 & 4,78 & 23,04 & 24,20 & 5,01 & 61,0 & 58,0 & 4,92 \\
\hline 2000 & 17,79 & 18,43 & 3,55 & 0,306 & 0,290 & 5,23 & 26,80 & 28,28 & 5,52 & 62,0 & 60,0 & 3,23 \\
\hline 2250 & 20,76 & 21,23 & 2,23 & 0,298 & 0,280 & 6,04 & 27,52 & 29,29 & 6,43 & 63,0 & 61,0 & 3,17 \\
\hline 2625 & 25,15 & 25,83 & 2,69 & 0,299 & 0,283 & 5,35 & 27,43 & 28,98 & 5,65 & 64,5 & 62,5 & 3,10 \\
\hline 3000 & 29,21 & 29,82 & 2,11 & 0,304 & 0,289 & 4,93 & 26,98 & 28,38 & 5,19 & 67,0 & 64,0 & 4,48 \\
\hline 3375 & 31,79 & 32,76 & 3,04 & 0,300 & 0,282 & 6,00 & 27,34 & 29,09 & 6,38 & 67,5 & 65,5 & 2,96 \\
\hline 3750 & 34,55 & 35,38 & 2,39 & 0,294 & 0,276 & 6,12 & 27,90 & 29,72 & 6,52 & 68,0 & 66,5 & 2,21 \\
\hline 4125 & 36,70 & 37,70 & 2,73 & 0,292 & 0,273 & 6,51 & 28,09 & 30,05 & 6,96 & 69,0 & 67,0 & 2,90 \\
\hline 4500 & 38,15 & 38,94 & 2,08 & 0,298 & 0,278 & 6,71 & 27,52 & 29,50 & 7,19 & 71,0 & 68,0 & 4,23 \\
\hline 4875 & 39,07 & 39,43 & 0,92 & 0,306 & 0,290 & 5,23 & 26,80 & 28,28 & 5,52 & 72,5 & 68,5 & 5,52 \\
\hline 5250 & 39,10 & 39,72 & 1,58 & 0,317 & 0,300 & 5,36 & 25,87 & 27,34 & 5,67 & 74,0 & 71,5 & 3,38 \\
\hline 5500 & 36,88 & 38,38 & 4,06 & 0,332 & 0,315 & 5,12 & 24,71 & 26,04 & 5,40 & 75,0 & 73,0 & 2,67 \\
\hline \multicolumn{2}{|c|}{ Min } & & 0,92 & & & 4,78 & & & 5,01 & & & 2,21 \\
\hline \multicolumn{2}{|c|}{ Max } & & 4,06 & & & 6,71 & & & 7,19 & & & 5,52 \\
\hline & & 30,89 & & 0,31 & 0,29 & & 26,67 & 28,26 & & 67,88 & 65,46 & \\
\hline \multicolumn{2}{|c|}{ Average increase } & $2,41 \%$ & \multicolumn{2}{|c|}{ Average decrease } & $5,59 \%$ & \multicolumn{2}{|c|}{ Average increase } & $5,98 \%$ & \multicolumn{2}{|c|}{ Average decrease } & $3,56 \%$ & \\
\hline \multicolumn{2}{|c|}{ *Informal values } & & & & & & & & & & & \\
\hline
\end{tabular}

Relative increase of Effective power $\left(P_{e}\right)$

[\%]

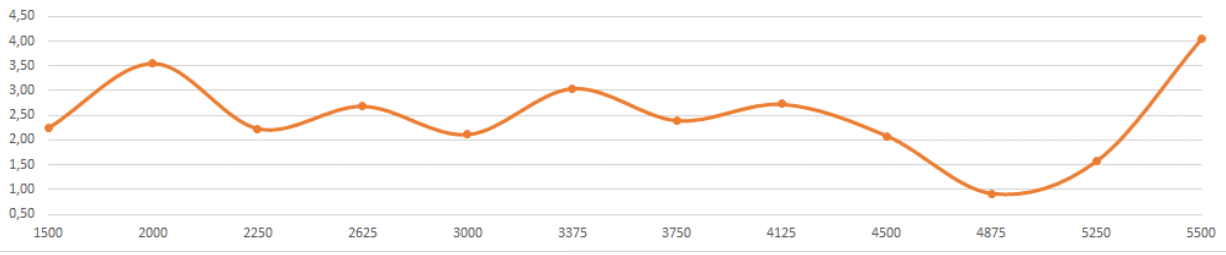

Fig. 3. Relative increase of Effective power (Pe).

Relative decrease of Actual specific consumption $\left(C_{e}\right)$

$[\%]$

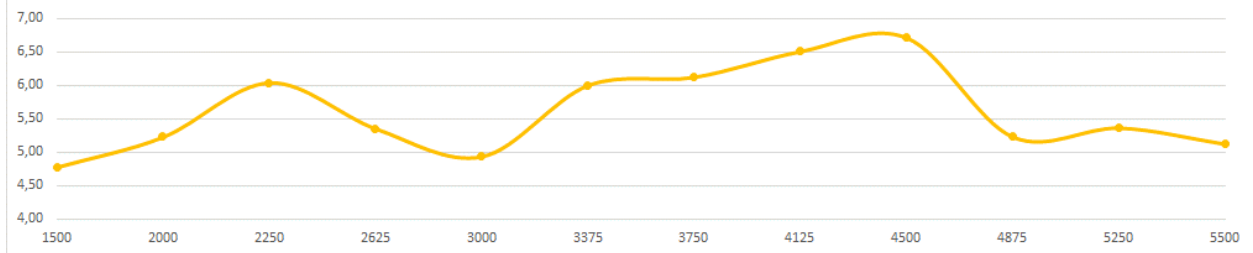

Fig. 4. Relative decrease of Actual specific consumption (Ce).

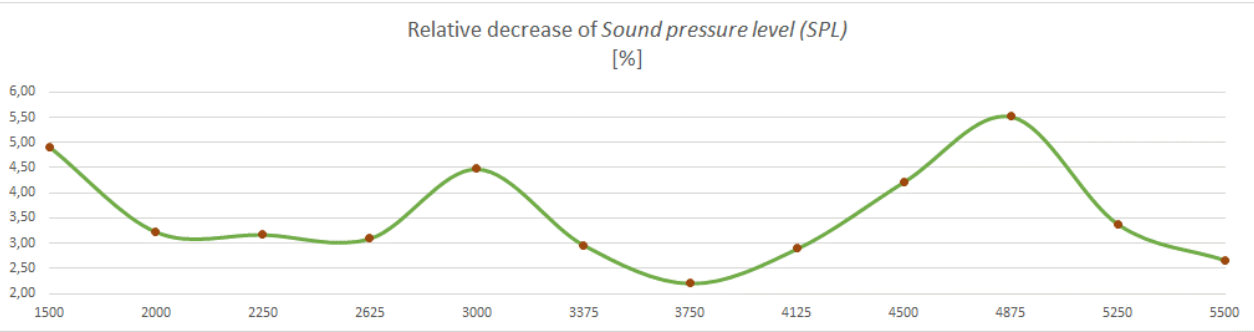

Fig. 5. Relative decrease of Sound pressure level (SPL). 
Results of analyse Modified engine vs. Standard engine:

i. Regarding the Effective power $\left(P_{e}\right)$, there is an increase between $0,92 \div 4,06 \%$, showing an increasing in the average value of $2,41 \%$;

ii. Actual specific consumption $\left(C_{e}\right)$, shows a decrease between $4,78 \div 6,71 \%$; the average value of this decrease is $5,59 \%$;

iii. The decrease of Sound pressure level (SPL) is between 2,21\% and 5,52\%, showing a decreasing in the average value of $3,56 \%$.

\section{Conclusion}

Using the ceramic materials in the construction of the combustion chamber of spark-ignition engines seems ideal in terms of mechanical strength and functional benefits. Their main shortcoming is the fragility to shocks and vibrations which led to the successful use of composite materials. By using ceramic materials, the temperature in the firing chamber increases by $150 \div 200 \mathrm{~K}$ which favours the firing process. In addition to increasing the actual power, the actual engine torque and the reduction of the actual specific fuel consumption, the noise and, not to be neglected, the chemical pollution are reduced. The replacement of metallic materials with ceramic ones leads to a decrease in the motor mass due to the lower density of ceramic materials. The next step is to redesign the cooling system by using a smaller radiator and implicitly by decreasing the volume of the coolant.

Future research will focus on redesigning the cooling system by using a smaller radiator (implicitly decreasing the volume of the coolant) and improving the current ceramic crown bonding solution on the piston surface.

\section{References}

1. N. Bataga, N. Burnete, I. Barabas, A. Cazila, N. Filip, A. Naghiu, F. Dan, R. Szasz, D. Karamusantas, Motoare cu ardere interna. Combustibili. Lubrifianti. Materiale speciale pentru autovehicule. Economicitate. Poluarel Internal combustion engines. Fuels. Lubricants. Special materials for vehicles. Economy. Pollution (U.T.Press, Cluj-Napoca, 2000)

2. G. Evangelos, Energies, 10(11), 1882, DOI: 10.3390/en10111882 (2017)

3. A. Chiru, H.R. Anca, C. Cofaru, R. Kuchar, A. Soica, N. Ispas, Materiale compozite. Vol.I/ Composite materials. Vol.I (E.U.T., Brasov, 1999)

4. N. Burnete, N. Bataga, D. Karamusantas, Constructia si calculul motoarelor cu ardere internal Construction and calculation of internal combustion engines (Todesco, ClujNapoca, 2001)

5. N. Burnete et al., Motoare diesel si biocombustibili pentru transportul urban/ Diesel engines and biofuels for urban transport (Mediamira, Cluj-Napoca, 2008)

6. D. Radu, R. State, Romanian Journal of Materials 41 3, 269 (2011)

7. I. Barabas, I.A. Todorut, DOI: 10.5772/25370 (2011)

8. N. Negurescu, C. Pana, M.G. Popa, Motoare cu aprindere prin scanteie-Procese/ Spark ignition engines-Processes (Matrixrom, Bucuresti 2009)

9. M.L. Scutaru, A. Chiru, S. Vlase, C. Cofaru, H. Teodorescu-Draghicescu, Materiale plastice si compozite in ingineria autovehiculelor/ Plastics and composites in automotive engineering (Matrixrom, Bucuresti, 2013) 